\title{
转基因骨髓基质细胞系对人脐血 $\mathrm{CD34}^{+}$ 细胞的体外扩增作用*
}

\author{
张 毅 ${ }^{* *}$ 唐佩弦 金 漟 李秀森 \\ 张双喜 吴英毛宁 \\ (军事医学科学院基础医学研究所, 北京 100850)
}

\begin{abstract}
摘要 为探讨转染 $F L$ 和/或 $T P O$ 基因骨髓基质细胞系对脐血 $\mathrm{CD} 34^{+}$细胞的体外扩增 效应，建立了转基因骨髓基质细胞系共培养体系。采用免疫磁珠法分离人脐血 CD $34^{+}$细 胞，在 CD34 ${ }^{+}$细胞不同体外培养体系中取样测试细胞总数、CD34 细胞百分率和 $\mathrm{CFC}($ 包 括 CFU-GM 和 BFU-E). 结果表明，在 8 种不同组合的培养体系中，转基因基质细胞共 培养体系较无基质液体培养体系对细胞总数, $\mathrm{CFC}, \mathrm{CD} 34^{+}$细胞均具有明显的扩增效应,

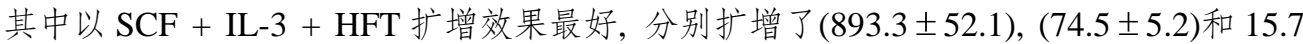
倍. CFU-GM 和 BFU-E 在第 2 周时达扩增高峰, 扩增倍数分别为(78.1 \pm 5.5$)$ 和(57.0土 19.7). LTC-IC 测定结果显示, 只有 SCF + IL-3 + FL + TPO 和 SCF + IL-3 + HFT 组有 LTC-IC 的存在, 统计学检验无显著性差异. 上述结果提示, 转基因骨髓基质细胞系可 通过细胞间的接触协同其他细胞因子增强对脐血 $\mathrm{CD} 34^{+}$细胞的体外扩增作用.
\end{abstract}

\section{关键词 体外扩增 CD34+细胞 基质细胞系 血小板生成素 Flt3 配基}

脐血含有大量的造血干/祖细胞, 至今已有 3000 多例以脐血移植治疗儿童恶性肿瘤和非恶 性肿瘤的成功范例. 但由于单份脐血造血细胞数量不足以满足成人移植需要, 因此需大量扩 增后才能使用. 许多研究结果表明, 血小板生成素(TPO)和 Flt3 配基(Flt3 ligand, FL)对早期造 血细胞的扩增具有明显的促进作用, 以基质细胞作为培养底层与外源细胞因子组成的共培养 体系对扩增 $\mathrm{CD}_{3} 4^{+}$细胞及保持其长期重建造血能力具有重要调控作用 ${ }^{[1,2]}$. 鉴于此, 本研究探 讨了转染 FL 和 TPO 的人骨髓基质细胞系对脐血 CD34 细胞的体外扩增作用.

\section{1 材料与方法}

\section{1 材料}

1.1.1 细胞系和脐血样品 转染 FL 的骨髓基质细胞系 HDF, 转染 TPO 的骨髓基质细胞系 HLT, 转染 $F L$ 和 $T P O$ 双基因的骨髓基质细胞系 HFT 均由张毅博士完成 ${ }^{[3]}$. 正常人骨髓基质细 胞系由中国医学科学院血液学研究所姜学英教授惠赠. 脐血取自北京协和医院妇产科. 磁性

2000-07-04 收稿, 2000-12-01 收修改稿

* 国家 “863”高科技重点课题基金资助项目(批准号: BH-030501)

** E-mail: jbteng@ sohu.com 
细胞分离仪和 $\mathrm{CD} 34^{+}$细胞分离试剂盒为德国 Miltenyi Biotec GmbH 公司产品.

\subsection{2 细胞因子和抗体重组人干细胞因子(rhSCF)由 Immunotec 公司赠送. 重组人 Flt3} 配基(FL)、重组人血小板生成素(TPO)、重组人粒-巨噬细胞集落刺激因子(rhGM-CSF)、重组 人白细胞介素(rhIL)-3、重组人粒系细胞集落刺激因子为 Peprotech 公司产品. 重组人红细胞生 成素(EPO)为军事医学科学院放射医学研究所产品. 抗 CD34 单克隆抗体(CD34-PE)和抗 CD38 单克隆抗体(CD38-FITC)均为 Pharmingen 公司产品.

\subsection{3 主要试剂 BSA, 甲基纤维素, $\beta$-颈基乙醇, L-谷氨酰胺, 氢化可的松等均为 Sigma} 公司产品. 细胞培养基、马血清为 GIBCO 公司产品. LTC-IC 专用培养基为 Stem Cell 公司产品.

\section{2 方法}

1.2.1 脐血的采集和 $\mathrm{CD}^{+}{ }^{+}$细胞的分离纯化 收集正常、健康、足月分娩胎儿的脐血于 含抗凝剂的采血袋中, $0 \sim 4^{\circ} \mathrm{C}$ 保存和运输, $4 \mathrm{~h}$ 内进行 $\mathrm{CD} 34^{+}$细胞的分离, 用 Ficoll/Hypaque(比重 为 1.077)密度离心分离脐血单个核细胞. 按磁性细胞分选仪和 $\mathrm{CD} 34^{+}$细胞分离试剂盒说明分 离纯化脐血 CD34 ${ }^{+}$细胞 ${ }^{[4]}$. 用抗 CD34 单克隆抗体和抗 CD38 单克隆抗体结合流式细胞仪计数 阳性细胞百分率.

1.2.2 扩增分组 实验分为无基质液体培养组和转基因基质细胞共培养组. 前者根据不同 因子组合分为: ( i ) SCF+ IL-3; (ii ) SCF + IL-3 + FL; (iii) SCF + IL-3 + TPO; (iV) SCF + IL-3 +FL+TPO. 后者根据不同基质细胞分为: ( v ) HFCL + SCF + IL-3; (vi) HDF + SCF+IL-3; (vii) $\mathrm{HLT}+\mathrm{SCF}+\mathrm{IL}-3$; (viii) HFT + SCF + IL-3.

\subsubsection{CD34 ${ }^{+}$细胞体外培养 $\mathrm{CD}^{+} 4^{+}$细胞体外液体培养体系为 $\mathrm{IMDM}$ 中含 $12.5 \% \mathrm{HS}$,} $12.5 \% \mathrm{BSA}, 2 \mathrm{mmol} / \mathrm{L} \mathrm{L}$-谷胺酰氨, $1 \times 10^{-4} \mathrm{~mol} / \mathrm{L} 2$-颈基乙醇, $1 \times 10^{-6} \mathrm{~mol} / \mathrm{L}$ 氢化可的松和不 同重组细胞因子. 各种细胞因子用量为： $\mathrm{rhSCF} 50 \mathrm{ng} / \mathrm{mL}, \mathrm{rhIL}-310 \mathrm{ng} / \mathrm{mL}, \mathrm{rhFL} 10 \mathrm{ng} / \mathrm{mL}$, rhTPO $10 \mathrm{ng} / \mathrm{mL}$. CD $34^{+}$细胞按 $1 \times 10^{4}$ 个 $/ \mathrm{mL}$ 接种于无或铺满 $50 \%$ 左右转基因基质细胞的 24 孔板中, 每孔 $1 \mathrm{~mL}, 37^{\circ} \mathrm{C}, 5 \% \mathrm{CO}_{2}$ 培养 4 周. 每周半量换液, 基质细胞层每周更换 2 次. 每周 进行细胞总数、 $\mathrm{CD} 34^{+}$细胞比例及各系造血祖细胞检测.

\subsection{4 造血祖细胞集落培养 $\quad \mathrm{CD} 34^{+}$细胞和不同培养体系扩增细胞在甲基纤维素体系中} 测定其集落形成能力. 接种细胞数分别为第 7 天 5000 个 $/ \mathrm{mL}$ ，第 14 天 $10^{4}$ 个/ $\mathrm{mL}$, 第 21 天 5 $\times 10^{4}$ 个 $/ \mathrm{mL}$ ，第 28 天 $10^{5}$ 个 $/ \mathrm{mL}$. 培养体系为：IMDM 中含 $30 \% \mathrm{HS}, 1 \% \mathrm{BSA}, 2 \mathrm{mmol} / \mathrm{L} \mathrm{L}$-谷 氨酰胺, $1 \times 10^{-4} \mathrm{~mol} / \mathrm{L} 2$-颈基乙醇, $1 \%$ 甲基纤维素和重组细胞因子. 细胞因子包括: rhSCF 10 $\mathrm{ng} / \mathrm{mL}$, rhIL-3 $10 \mathrm{ng} / \mathrm{mL}$, rhGM-CSF $10 \mathrm{ng} / \mathrm{mL}$, rhG-CSF $10 \mathrm{ng} / \mathrm{mL}$, rhEPO 4 U/mL. 所有培养均在 24 孔培养板中进行, $37{ }^{\circ} \mathrm{C}, 5 \% \mathrm{CO}_{2}$, 饱和湿度条件下培养 $14 \mathrm{~d}$. 在倒置显微镜下直接计数 CFU-GM 和 BFU-E, 大于 50 个细胞的作为一个集落.

1.2.5 LTC-IC 培养使用 Dexter 方法将正常骨髓基质细胞经 10 15 Gy ${ }^{60}$ Co $\gamma$ 射线照射 后作为培养底层, 将培养 4 周后的各组造血细胞按一定的比例接种于培养底层上, 继续培养 4 周, 每周取出三复孔进行造血祖细胞集落培养, 计数产生的集落数, 培养第 5 8 周所产生的集 落总和为 LTC-IC 总数.

\subsection{6 统计学处理 采用 Student $t$ 检验评定差异的显著性.}




\section{2 结果}

\section{1 脐血 $\mathrm{CD}^{+} 4^{+}$细胞的分离和纯化}

用 MACS 系统可获得高纯度的 CD34 ${ }^{+}$细胞, 经 FACS 检测, 纯度可达 $95.0 \%, \mathrm{CD}^{2} 4^{+} \mathrm{CD} 38^{-}$ 细胞纯度为 $10.0 \%$ (图 1).
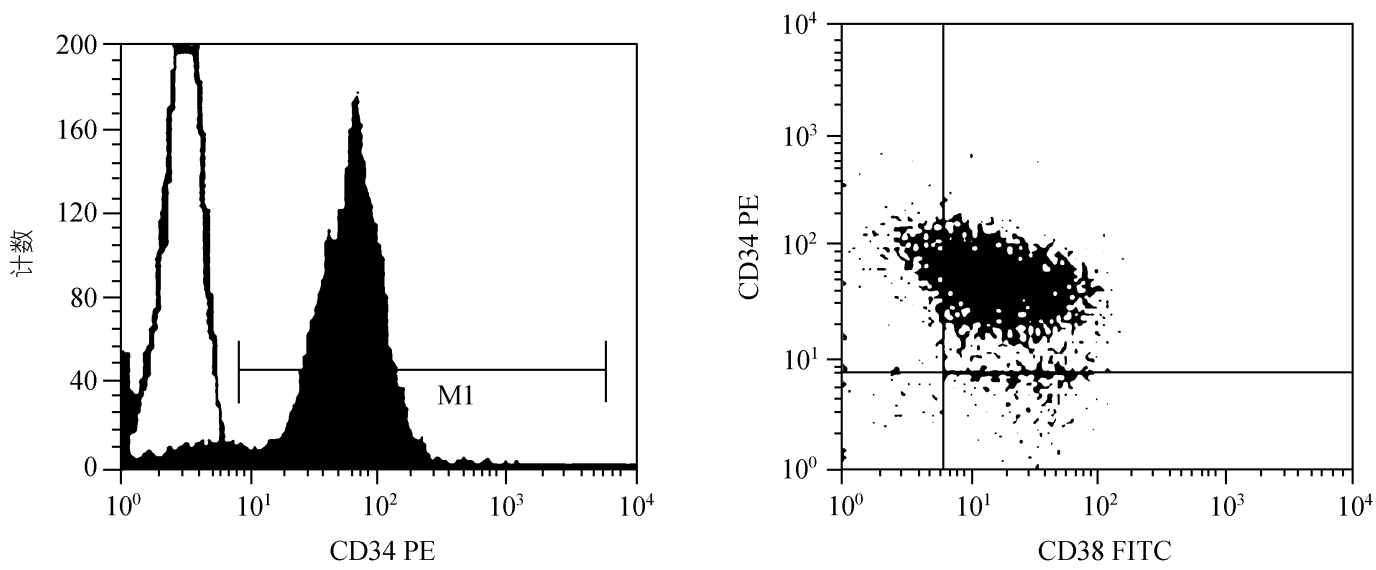

图 1 流式细胞仪测定磁性细胞分离仪分离的人脐血 CD34+细胞的纯度

\section{2 转基因基质细胞对细胞总数, CFC, CFU-GM 和 BFU-E 的扩增作用}

根据重组细胞因子和转基因基质细胞对 $\mathrm{CD} 34^{+}$细胞的作用特点, 分为 8 组不同组合, 并连 续观察 4 周. 在含细胞因子的无基质液体培养组的培养过程中发现, 细胞开始时沿培养板的边 缘生长, 并逐渐向培养板中心铺开, 而在转基因基质细胞共培养组的培养时, 细胞向有基质细 胞区聚集生长的现象. 第 1 周各组细胞总数均有增加; 第 2 4 周时转基因基质细胞共培养组的 细胞总数均比无基质液体培养组明显增加 $(P \leqslant 0.05)$; 培养至第 4 周时, SCF + IL-3 + HFT 组细 胞总数增加量最大, 扩增倍数达到 $893.3 \pm 52.1$ (表 1 ).

表 1 脐血造血细胞总数的扩增结果

\begin{tabular}{ccccc}
\hline \multirow{2}{*}{ 组别 } & \multicolumn{3}{c}{ 培养时间 / d } \\
\cline { 2 - 5 } & 7 & 14 & $331.3 \pm 25.3$ & $624.0 \pm 42.3$ \\
SCF + IL-3 & $26.0 \pm 1.0$ & $152.7 \pm 2.1$ & $376.7 \pm 18.6$ & $710.7 \pm 88.8$ \\
SCF + IL-3 + FL & $37.8 \pm 1.0$ & $188.0 \pm 9.0$ & $367.3 \pm 26.4$ & $694.7 \pm 58.3$ \\
SCF + IL-3 + TPO & $33.3 \pm 2.1$ & $176.7 \pm 7.1$ & $428.0 \pm 25.0$ & $762.7 \pm 72.6$ \\
SCF + IL-3 + FL + TPO & $52.0 \pm 6.6$ & $215.0 \pm 7.8$ & $340.7 \pm 57.1$ & $638.7 \pm 17.2$ \\
SCF + IL-3 + HFCL & $31.8 \pm 1.9$ & $166.4 \pm 2.6$ & $394.0 \pm 37.5$ & $796.0 \pm 52.5$ \\
SCF + IL-3 + HDF & $46.8 \pm 6.8$ & $191.6 \pm 8.6$ & $390.7 \pm 28.1$ & $764.0 \pm 62.9$ \\
SCF + IL-3 + HLT & $43.0 \pm 1.0$ & $183.7 \pm 21.2$ & $474.7 \pm 16.2$ & $893.3 \pm 52.1$ \\
SCF + IL-3 + HFT & $64.2 \pm 3.4$ & $228.3 \pm 6.7$ &
\end{tabular}

在 4 周的体外培养中, 8 组不同组合对 CFC 产率具有不同的扩增效率 $(P \leqslant 0.05)$. 扩增倍数 在第 2 周时达到峰值, 其中 SCF + IL-3 + HFT 组扩增倍数高达 $74.5 \pm 5.2$, 其次为 SCF + IL-3 + $\mathrm{FL}+\mathrm{TPO}$ 组, 扩增倍数为 $60.8 \pm 5.8$, 其他 6 组数据中转基因组扩增的倍数均显著高于无基质 液体培养组(图 2). 


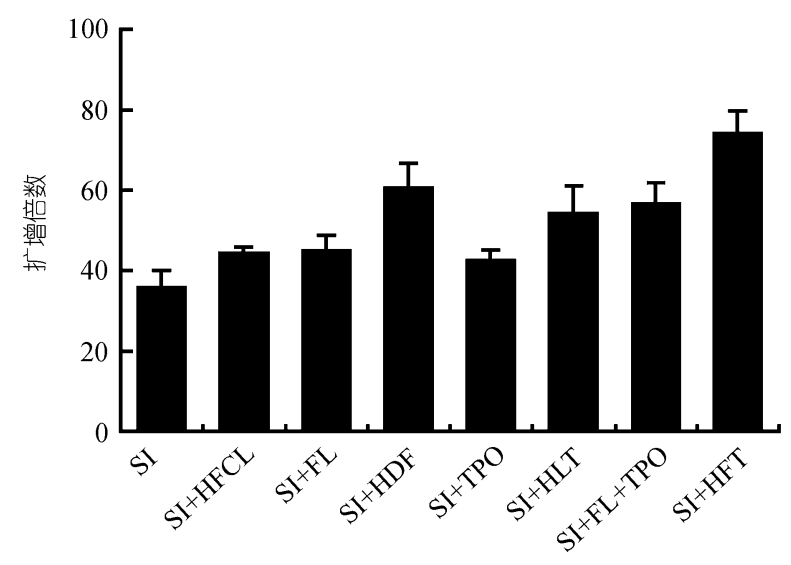

图 2 不同组合体外培养第 2 周时 $\mathrm{CFC}$ 扩增倍数的变化 $\mathrm{SI}=\mathrm{SCF}+\mathrm{IL}-3 ; \mathrm{CFC}=\mathrm{CFU}-\mathrm{GM}+\mathrm{BFU}-\mathrm{E}$
每周取培养体系中的悬浮细胞(造血 细胞)做 CFU-GM 和 BFU-E 测定, 结果表 明, 第 1 周时, 转基因基质细胞共培养组 CFU-GM 和 BFU-E 的数量均较无基质液 体培养组有轻度增加. 第 2 周时, 各组 CFU-GM 和 BFU-E 增加显著, 尤以 SCF + IL-3 + HFT $($ SI + HFT) 组增加最明显, 分 别扩增了 $(78.1 \pm 5.5)$ 和 $(57.0 \pm 19.7)$ 倍, 以后随着扩增时间的延长, 造血细胞集 落的产率逐渐降低, 集落体积也逐步减 少(数据略). 上述结果说明, 转基因基质 细胞在体外协同其他细胞因子可促进造 血干/祖细胞的增殖.

\section{3 转基因基质细胞对脐血 $\mathrm{CD34}^{+}$细胞的扩增作用}

CD 34 细胞培养至第 7, 14, 28 天时, 分别取样用 PE-CD34 单克隆抗体和 FITC-CD38 单克 隆抗体标记, FACS 测试其中 $\mathrm{CD} 34^{+}$细胞的百分率和 $\mathrm{CD} 34^{+} \mathrm{CD} 38^{-}$细胞的百分率, 以未标记的 细胞作为对照. 结果表明, CD $34^{+}$细胞总数在第 1 周扩增以 SCF + IL-3 + HFT $(\mathrm{SI}+\mathrm{HFT})$ 组和 $\mathrm{SCF}+\mathrm{IL}-3+\mathrm{FL}+\mathrm{TPO}(\mathrm{SI}+\mathrm{FT})$ 组最显著, 分别扩增了 15.7 和 12.1 倍. 此峰值在第 2 周就降至 3.9 和 2.7 倍, 第 4 周时 $\mathrm{CD} 34^{+}$细胞仅为 0.8 和 0.4 倍. 而 $\mathrm{CD} 34^{+} \mathrm{CD} 38^{-}$细胞在第 2,4 周的扩增 倍数分别为 16 和 $28,1.5$ 和 5.3, 提示 FL 和 TPO 对早期祖细胞的扩增作用显著, 但机制尚未 完全清楚(表 2).

表 $2 \mathrm{CD}^{2} 4^{+}$细胞和 $\mathrm{CD} 34^{+} \mathrm{CD} 38^{-}$细胞体外培养 4 周细胞扩增倍数的变化

\begin{tabular}{|c|c|c|}
\hline \multirow{2}{*}{ 培养时间 / d } & $\mathrm{SCF}+\mathrm{IL}-3+\mathrm{FL}+\mathrm{TPO}$ & SCF + IL-3 +HFT \\
\hline & $\mathrm{CD}^{2} 4^{+} / \mathrm{CD} 34^{+} \mathrm{CD} 38^{-}\left(1 \times 10^{4}\right)$ & $\mathrm{CD}^{2} 4^{+} / \mathrm{CD} 34^{+} \mathrm{CD} 38^{-}\left(1 \times 10^{4}\right)$ \\
\hline 0 & 0 & 0 \\
\hline 7 & $12.1 /-$ & $15.7 /-$ \\
\hline 14 & $2.7 / 16$ & $3.9 / 28$ \\
\hline 28 & $0.4 / 1.5$ & $0.8 / 5.3$ \\
\hline
\end{tabular}

\section{4 转基因基质细胞层对 LTC-IC 的支持作用}

LTC-IC 是一群很早期的造血祖细胞, 它们从增殖、分化到形成集落所需要的时间比相对 成熟的造血干细胞要长得多, 在长期培养中至第 5 周时才充分形成集落, 因此 LTC-IC 被定为 长期培养第 5 周以后产生的集落. 我们的实验显示, 在 8 组不同组合培养 4 周后, 将悬浮细胞 和贴壁细胞混匀, 接种于 ${ }^{60} \mathrm{Co} \gamma$ 射线照射的骨髓基质细胞层上继续培养 4 周, 每周测定 LTC-IC 的产率, 结果表明, 只有两组即 SCF + IL-3 + FL + TPO 和 SCF + IL-3 + HFT 组在第 5 6 周产生 LTC-IC, 其总和分别为 $3.0 \pm 0.0$ 和 $4.5 \pm 2.1$, 统计学检验无显著性差异. 鉴于 HFT 为转 染有 $F L$ 和 $T P O$ 双基因的骨髓基质细胞系, 这一结果支持 FL 和 TPO 协同作用对早期造血具 有支持作用的观点. 


\section{3 讨论}

脐血作为骨髓造血干/祖细胞以外的一种来源, 自 1988 年用于治疗 Fanconi 贫血以来, 已 日益广泛应用于临床. 脐血移植的最佳细胞剂量为 $(3 \sim 5) \times 10^{5}$ 个 $/ \mathrm{kg}$ 体重, 这样就使其在成人 移植中的临床应用受到了限制, 因而扩增脐血 $\mathrm{CD} 34^{+}$细胞成为目前研究的热点. 近年来随着 对造血微环境的深人研究, 发现造血微环境是造血干/祖细胞(CD34+细胞)生存所必需的体内 环境, 其调节造血的机制是通过骨髓基质细胞与造血干/祖细胞的密切接触以及造血生长因子 的作用完成的 ${ }^{[5 ~ 7]}$. 已有研究证实, 骨髓基质细胞作为造血微环境中的主要成分, 对造血干细 胞的自我更新, 迁移与调亡, 各系祖细胞的发育、分化与成熟等均具有十分重要的调控作用 ${ }^{[8,9]}$. Aiuti 等人 ${ }^{[10]}$ 研究表明, 在有基质细胞支持的共培养体系中, 前 4 周可明显扩增 CD34 ${ }^{+}$细胞并 在体外维持 $\mathrm{CD} 34^{+}$细胞存在达 10 周左右. Kawada 等人 ${ }^{[11]}$ 发现, 在与基质细胞共培养的体系中 加人早期造血刺激因子 FL 和 TPO, 可明显扩增冻存的脐血 $\mathrm{CD} 34^{+} \mathrm{CD} 38^{-}$细胞, $7 \mathrm{~d}$ 即可达到 100 倍的扩增效应, CFC, CFU-GEMM 和 LTC-IC 分别扩增 10 30 倍, 10 20 倍和 25 倍. 在对转 基因基质细胞体外扩增脐血 CD34 细胞的研究中, 得出以下结果: (i ) 在利用 MACS 系统获 得高纯度脐血 CD $34^{+}$细胞的基础上, 经 SCF, IL-3, FL, TPO 与转基因基质细胞的不同组合培养 4 周, 可不同程度地扩增细胞总数、 $\mathrm{CFC}$ 及 $\mathrm{CD} 34^{+}$细胞. 转基因基质细胞组较无基质细胞液体 培养组的造血细胞总数和 CFC 明显增多, 细胞总数扩增倍数最高可达 $893.3 \pm 52.1$. CFC 扩增 在第 2 周时达到峰值, 其中 SCF + IL-3 + HFT 组扩增倍数高达 $74.5 \pm 5.2$, 显著高于单基因组 及无基质细胞液体培养组 $(P \leqslant 0.05)$, 但随着培养时间的延长, $\mathrm{CFC}$ 产率逐步减少. 不同培养体 系中造血细胞产生 CFU-GM 和 BFU-E 的能力不同, 转基因基质细胞共培养组 CFU-GM 和 BFU-E 的数量较无基质细胞液体培养组均有增加. 第 2 周时扩增达到峰值, 尤以 SCF + IL-3+ HFT 组增加最明显. (ii ) 在对 $\mathrm{CD} 34^{+}$细胞及 $\mathrm{CD} 34^{+} \mathrm{CD} 38^{-}$细胞扩增作用的动态观察中, 发现 $\mathrm{SCF}+\mathrm{IL}-3+\mathrm{HFT}$ 组扩增作用最强, CD34 细胞总数在第 1 周达峰值即扩增了 15.7 倍, 此后逐 步降低; $\mathrm{CD} 34^{+} \mathrm{CD} 38^{-}$细胞在第 2 周时扩增了 28 倍, 第 4 周时为 5.3 倍, 此结果说明转基因基 质细胞对 $\mathrm{CD} 34^{+}$细胞体外培养在短期内的扩增作用明显, 且以 FL + TPO 组合对早期造血祖细 胞具有较好的扩增效果并可维持部分 $\mathrm{CD} 34^{+}$细胞亚群存活达 4 周左右, 其机理有待进一步深 人探讨. 以上结果说明, 转基因基质细胞在体外可协同其他细胞因子促进 $\mathrm{CD} 34^{+}$细胞的扩增 作用及 CFU-GM 和 BFU-E 的增殖. 不同细胞因子组合及是否存在转基因基质细胞, 对 CD $34^{+}$ 细胞扩增效果明显不同, 适宜的组合可达到短期高效扩增的目的, 并可保持部分早期造血祖 细胞.

在体外完成短期扩增 $\mathrm{CD} 34^{+}$细胞后, 需要对扩增的 $\mathrm{CD} 34^{+}$细胞是否在体内维持长期造血 重建进行深人研究. 已有研究表明, 体内长期造血重建与长期培养起始细胞(LTC-IC)的含量有 关 ${ }^{[12,13]}$. LTC-IC 作为一群至今为止经体外培养而获得的最原始的造血祖细胞, 对它的特征研 究和含量测定可为预测造血干细胞移植能否获得成功提供客观指标. 在进行 LTC-IC 的研究 时, 我们采用将体外长期共培养 4 周的不同组合的造血细胞, 移种到经 ${ }^{60} \mathrm{Co} \gamma$ 射线照射的正常 人骨髓基质细胞层上, 进行为期 4 周的 LTC-IC 培养. 结果显示, 只有两组即 SCF + IL- $3+\mathrm{FL}+$ $\mathrm{TPO}$ 和 SCF + IL-3 + HFT 组有 LTC-IC 的存在. 鉴于 HFT 为转染有 $F L$ 和 TPO 双基因的骨髓 基质细胞系，这一结果进一步证明 FL 和 TPO 协同作用对早期造血具有支持作用. 
致谢 感谢北京协和医院妇产科潘凌亚副教授、童英博士、朱噃丽硕士为本研究提供脐 血样本.

\section{参 考文 献}

1 Luens K M, Travis M A, Chen B P, et al. Thrombopoietin, kit ligand, and flk2/flt3 ligand together induce increased numbers of primitive hematopoietic progenitors from human $\mathrm{CD} 34^{+} \mathrm{Thy}^{+} \mathrm{Lin}^{-}$cells with preserved ability to engraft SCID-hu bone. Blood, 1998, 91: 1206 1215

2 Piacibello W, Sanavio F, Garetto L, et al. Extensive amplification and self-renewal of human primitive hematopoietic stem cells from cord blood. Blood, 1997, 89(8)L: 2644 2653

3 张 毅, 唐佩弦, 李秀森, 等. 应用 IRES 序列研究人 FL 与 TPO 基因在转染 HFCL 细胞中的表达. 中华血液学杂志, 1999, 12(20): 624 627

4 Zhou S, Mao N , Zhao M , et al. Effect of recombinant FLT3 ligand (rhFL) on in vitro expansion of human cord blood CD34 cells. Natl Med J China, 1998, 78: 37 39

5 Dexter T M, Allen T D, Lajtha L G, et al. Conditions controlling the proliferation of hematopoietic stem cells in vitro. J Cell Physiol, 1997, 91: 335 344

6 Petzer A L, Hogge D E, Lansdorp P M, et al. Self-renewal of primitive human hematopoietic cells (long-term-cultureintiating cells) in vitro and their expansion in defined medium. Pro Natl Acad Sci USA, 1996, 93: 1470 1474

7 Sirchia G, Rebulla P. Palcental/umbilical cord blood transplantation. Haematologica, 1999, 84: 738 747

8 Wineman J, Moore K, Lemischka I, et al. Functional heterogeneity of the hematopoietic microenvironment: Rare stromal elements maintain long-term repopulating stem cells. Blood, 1996, 87: 4082 4090

9 Szilvassy S J, Weller K P, Lin W, et al. Leukemia inhibitory factor up-regulates cytokine expression by a murine stromal cell line enabling the maintenance of highly enriched competitive repopulating stem cells. Blood, 1996, 87: 4618 4628

10 Aiuti A, Fredrich C, Sieff C A, et al. Identification of distinct elements of the stromal microenvironment that control human hematopoietic stem/progenitor cell growth and differentiation. Exp Hematol, 1998, 26: 143 157

11 Kawada H, Ando K, Tsuji T, et al. Rapid ex vivo expansion of human umbilical cord hematopoietic progenitors using a novel culture system. Exp Hematol, 1999, 27: 904 915

12 Alcorn M J, Holyoake T L, Richmond L, et al. CD34 ${ }^{+}$cells isolated from cryopreserved peripheral-blood progenitor cells can be expanded ex vivo and used for transplantation with little or no toxicity. J Clin Oncol, 1996, 14: 1839 1847

13 Van Hennik P B, Breems D A, Withagen C M, et al. Graft-failure can be predicted by testing the ability of stem cells to produce progenitors in long-term stroma-supported culture. Exp Hematol, 1997, 25: 742 